\title{
Modelo de aplicación de un Ciclo de Mejora del Aula (CIMA) en la asignatura Investigación Comercial (Grado de Administración y Dirección de Empresas)
}

\section{Application model of a Classroom Improvement Cycle (CIMA) in the Business Research course (Degree in Business Administration and Management)}

Pablo Ledesma Chaves.

ORCID: https://orcid.org/0000-0001-7058-4820

Universidad de Sevilla.

Departamento de Administración de

Empresas y Marketing. pledesma@us.es

DOI: http://dx.doi.org/10.12795/9788447231003.037

Pp.: 777-798 


\section{Introducción}

El presente documento describe la aplicación de un ciclo de mejora en el aula (CIMA) realizado en la asignatura "Investigación Comercial" perteneciente al cuarto curso del grado Administración y Dirección de Empresas. La duración del CIMA fue de ocho horas, y correspondió a los temas dos y tres del temario propuesto por la asignatura durante el curso 2020-2021, concretamente a los temas referidos como "Definición del problema de investigación" y "Diseño de la investigación" respectivamente. El trabajo presenta particularidades específicas de adaptación de los contenidos, la metodología y la evaluación debido a las especiales circunstancias concurrentes por motivos de las restricciones provocadas por la pandemia del COVID19. Dentro de la asignatura, supuso un proceso innovador consultado y aplicado posteriormente por otros compañeros y que recibió el reconocimiento de los alumnos, y un alto grado de implicación.

\section{Descripción del contexto}

El CIMA de ocho horas tuvo lugar entre el veinte de octubre y el 4 de noviembre de 2020, dividido en cuatro sesiones de dos horas. Los estudiantes de la asignatura "Investigación comercial" de cuarto curso del grado de administración de empresas tenían una media de edad de 22 años, y estuvieron en todo momento informados del proceso, lo que favoreció su participación e implicación. El aula, concretamente el número 31 de la facultad de ciencias económicas, está preparada para 80 estudiantes, aunque por motivos de las restricciones sanitarias solo podían acudir a clase de manera presencial un tercio de esa cantidad. Los estudiantes tenían una rotación del $50 \%$ de presencialidad sobre los matriculados, por los que el resto debían seguir la clase a través de la plataforma virtual "BlackBoard Learn" de manera remota. Las restricciones

Ciclos de Mejora en el Aula (2020). Experiencias de Innovación Docente de la US Esta obra se distribuye con la licencia Creative Commons 
planteadas por la pandemia del COVID19 presentan importantes limitaciones a la aplicación del CIMA en cuanto a la realización de las actividades. No obstante, en todo momento se ha pretendido que todos los alumnos pudieran participar de manera activa en la experiencia.

\section{Diseño previo del CIMA}

\section{Mapa de contenidos y problemas clave}

El contenido del CIMA se ajustó a los temas dos y tres del temario de la asignatura para el curso 2020-2021, concretamente a los titulados "Definición del problema de investigación de mercados y desarrollo del enfoque" (Tema 2) y "Diseño de la investigación, datos secundarios y sindicados" (Tema 3), los cuales se encuentran encuadrados en el problema general de desarrollo de una investigación comercial para una empresa. El mapa de contenidos utilizado es el expresado en la Figura 1. El hilo conductor del CIMA sería un caso práctico sobre la empresa Harley-Davidson (Anexo 1), donde se plantea un problema empresarial de carácter estratégico, concretamente decidir si la empresa debe incrementar su volumen de producción de acuerdo a una demanda latente. Era conveniente para el planteamiento del CIMA que los alumnos comprendieran las especiales características de la empresa.

El mapa de contenidos, o el CIMA en general, comienza con una pregunta atrayente “¿Os gustan las motos?", para captar la atención, a partir de la cual todo el mapa de contenidos se articula sobre cuatro preguntas principales, destacadas en el mapa con un fondo arlequinada. La primera de ellas (“¿fabrico más motos?"), tiene la intención de expresar la dicotomía existente cuando se plantea un

Ciclos de Mejora en el Aula (2020). Experiencias de Innovación Docente de la US Esta obra se distribuye con la licencia Creative Commons 
problema empresarial contando con una visión por parte de la gerencia y otra por parte del investigador, por lo que deriva hacia dos ópticas diferentes que deben ponerse de acuerdo, siempre bajo el consejo profesional del investigador, y cómo armonizar ambos planteamientos. Esta visión conecta con la segunda pregunta básica (“¿Hay acuerdo entre ambas visiones? ¿Debe haberlo?") donde posicionamos al investigador como la figura relevante del tema, y donde una vez que está fijado, podemos comenzar con el diseño inicial de la investigación, o en su caso, equilibrar las visiones aportando los diferentes sintomas y las causas subyacentes. A partir de ese equilibrio, podemos comenzar con el enfoque de la investigación.

El enfoque parte de la tercera pregunta (“CCómo planteamos la investigación?"), y conocer el armazón derivado del diseño, donde concluimos planteando qué clase de conclusiones queremos alcanzar. Este planteamiento hace que cobre sentido la cuarta pregunta ("De acuerdo a las conclusiones que quieres llegar, ¿cómo las clasificarías?"), que determina la clase de datos que deben utilizar para proponer el diseño.

El esfuerzo en el desarrollo del modelo metodológico posible trató de pasar de un modelo tradicional transmisivo, hacia otro hipotético-deductivo, de manera que los conocimientos fueran adquiridos a través de la práctica (De Alba-Fernández y Porlán-Ariza, 2017), o la interpretación de casos reales, sin recurrir a la transmisión teórica salvo para alguna de las actividades de contraste, y que sea más duradero en el tiempo (Mora, 2017). Queríamos cambiar hacia un modelo donde el docente asumiera un papel de guía y apoyo en la transmisión de conocimientos (Finkel, 2008)

Ciclos de Mejora en el Aula (2020). Experiencias de Innovación Docente de la US Esta obra se distribuye con la licencia Creative Commons 

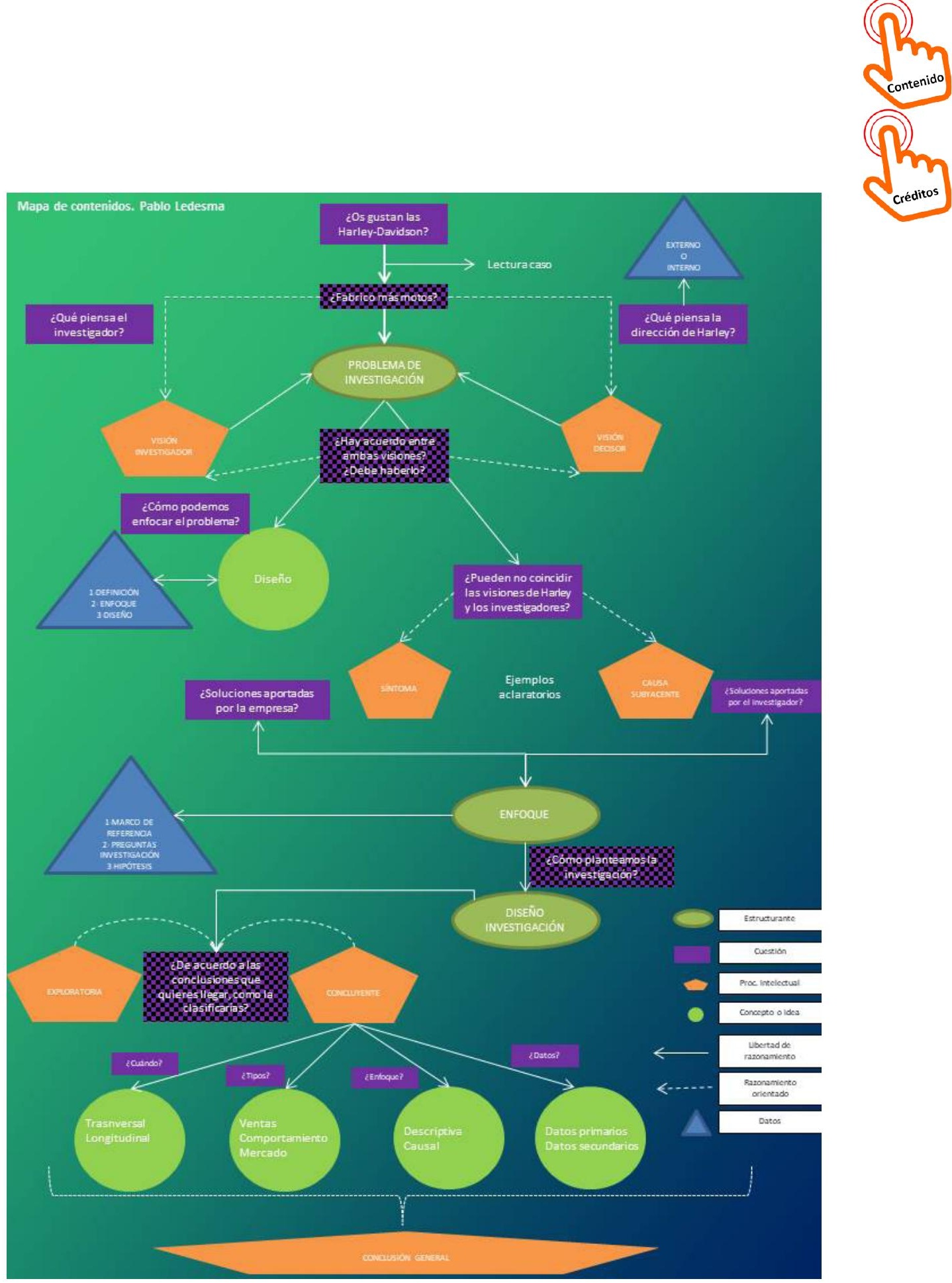

Figura 1. Mapa de contenidos

Ciclos de Mejora en el Aula (2020). Experiencias de Innovación Docente de la US (c) () $\odot$ Esta obra se distribuye con la licencia Creative Commons BY NC ND

Reconocimiento-NoComercial-SinObraDerivada Internacional (CC BY-NC-ND 4.0.) 


\section{Modelo metodológico, secuencia de actividades y cuestionario de ideas}

Las clases se desarrollarían en función de la exposición de un caso práctico como hilo conductor, que se retomaría en cada una de las sesiones añadiéndole nuevas partes al texto hasta completarlo de acuerdo a las necesidades de cada sesión y el seguimiento del mapa de contenidos. Posteriormente, se plantearía un trabajo o cuestión para que los alumnos, de manera individual o colectiva, comenzaran a desarrollarlo, aportando después una actividad de contraste para aclarar sus ideas y superar los obstáculos mentales. Tras ello, propondríamos otra actividad, relacionada o no con el caso, de manera que sirviera como asociación entre la realidad y el propio caso práctico, siguiendo de nuevo otra actividad de contraste, más participativa, para terminar con un pequeño mapa de contenidos en la pizarra con las aportaciones realizadas por los alumnos y su interrelación. Con este modelo metodológico hemos buscado el protagonismo total del estudiante (Bain, 2007).

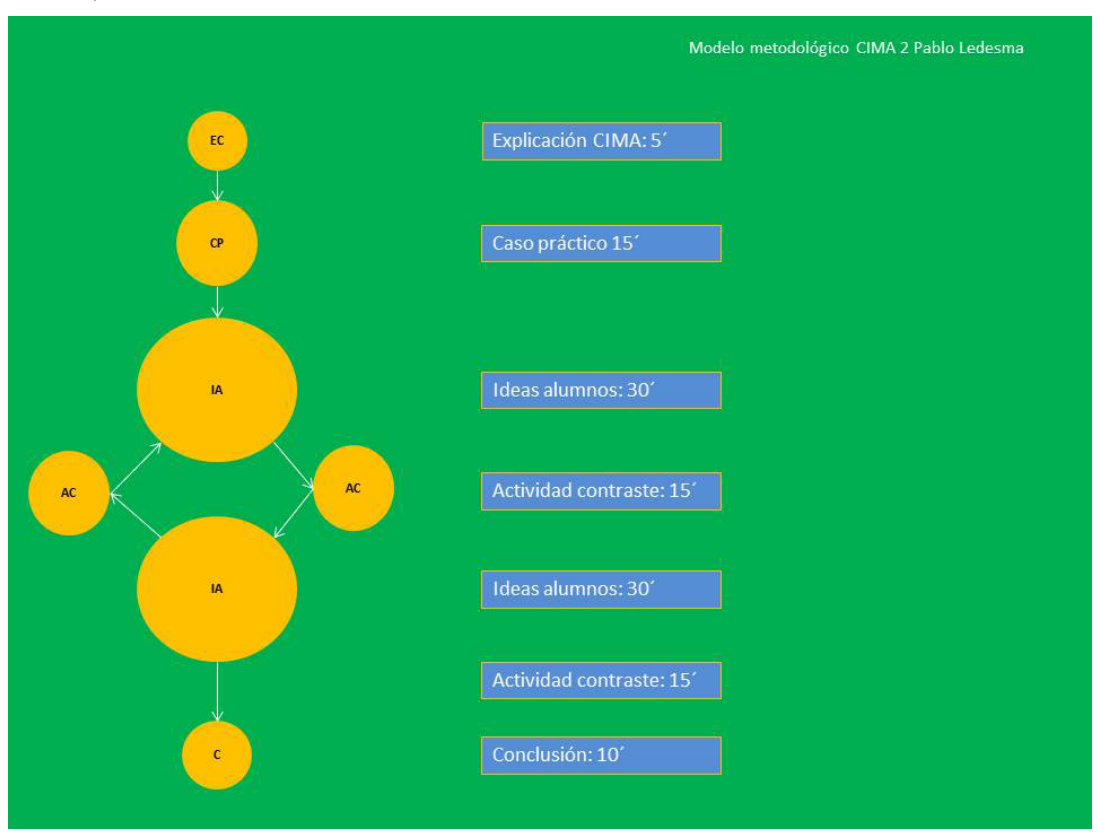

Figura 2. Modelo metodológico posible Ciclos de Mejora en el Aula (2020). Experiencias de Innovación Docente de la US
Esta obra se distribuye con la licencia Creative Commons Reconocimiento-NoComercial-SinObraDerivada Internacional (CC BY-NC-ND 4.0.) 
De manera previa al inicio del CIMA fue distribuido el cuestionario inicial (Tabla 2) de evaluación a través de la plataforma BlackBoard Learn a todos los alumnos matriculados en el grupo 4 de la asignatura, obteniéndose respuesta por parte de 40 alumnos, los cuales se convirtieron en 38 en el cuestionario distribuido tras la aplicación del CIMA. El cuestionario de evaluación inicial fue acompañado de un correo electrónico explicando las circunstancias del mismo, su importancia y su confidencialidad.

Las actividades propuestas en el desarrollo del CIMA (Tabla 1), en total ocho (dos por sesión), fueron planteadas de acuerdo al modelo metodológico y la secuencia de contenidos. Por el carácter de la publicación, exponemos a modo de ejemplo las correspondientes a la primera sesión, analizando después su escalera de aprendizaje.

Tabla 1. Secuencia de actividades del CIMA

\begin{tabular}{|c|l|c|}
\hline \multicolumn{2}{|c|}{$\begin{array}{c}\text { Sesión 1: Distinción entre problema de decisión administrativa y problema } \\
\text { de investigación de mercados. ¿Hay acuerdo entre ambas visiones? }\end{array}$} \\
\hline Actividad & Descripción & Tiempo \\
\hline $\begin{array}{c}\text { Explicación } \\
\text { CIMA }\end{array}$ & $\begin{array}{l}\text { Presentación del enfoque de las clases } \\
\text { correspondientes al CIMA. Explicación de la } \\
\text { metodología de trabajo, contenidos y evaluación. } \\
\text { Llamamiento a la participación activa. }\end{array}$ & $5 \mathrm{~m}$ \\
\hline Caso & $\begin{array}{l}\text { ¿Os gustan las motos? Planteamiento del caso } \\
\text { práctico Harley-Davidson. Lectura individual del } \\
\text { mismo, aunque previamente ha sido colgado } \\
\text { en la plataforma. Análisis de las especiales } \\
\text { características de la empresa, debido a sus } \\
\text { vínculos emocionales, así como su posición } \\
\text { competitiva privilegiada. Aportación de ideas por } \\
\text { parte de los alumnos y escritura en la pizarra por } \\
\text { mi parte. }\end{array}$ & $15 \mathrm{~m}$ \\
\end{tabular}

Ciclos de Mejora en el Aula (2020). Experiencias de Innovación Docente de la US Esta obra se distribuye con la licencia Creative Commons 


\begin{tabular}{|c|l|c|}
\hline $\begin{array}{c}\text { Ideas } \\
\text { Alumnos }\end{array}$ & $\begin{array}{l}\text { Actividad individual. Listado de ideas para trabajar } \\
\text { sobre un problema empresarial estratégico, } \\
\text { aplicado al caso de Harley Davidson. Cómo enfocar } \\
\text { la situación. Supone una interpretación más } \\
\text { extensa del cuestionario de evaluación. Exposición } \\
\text { oral de las ideas principales por parte de los } \\
\text { alumnos. Las ideas se anotan en la pizarra. }\end{array}$ & $30 \mathrm{~m}$ \\
\hline Actividad de \\
contraste 1 & $\begin{array}{l}\text { Indicación teórica, mediante la respuesta a las } \\
\text { siguientes preguntas presente en el mapa de } \\
\text { actividades: ¿Dónde acude la empresa a realizar su } \\
\text { investigación? ¿Debe ser interna o externa? ¿Qué } \\
\text { piensan ambas visiones? Ejemplo acerca de otra } \\
\text { visión dual del problema aplicado a otra empresa. }\end{array}$ & $15 \mathrm{~m}$ \\
\hline Ideas de los \\
alumnos & $\begin{array}{l}\text { Esquema o cuadro de dos columnas con ideas } \\
\text { sobre cómo detectarían el problema Harley- } \\
\text { Davidson y los investigadores }\end{array}$ & $30 \mathrm{~m}$ \\
\hline Actividad de \\
contraste 2 & $\begin{array}{l}\text { Exposición de casos reales en que la división } \\
\text { de opiniones entre empresa e investigador ha } \\
\text { supuesto un fracaso empresarial por la falta de } \\
\text { identificación correcta del problema. }\end{array}$ & $15 \mathrm{~m}$ \\
\hline Conclusión & $\begin{array}{l}\text { Puesta en común de ideas. Orientación hacia } \\
\text { el planteamiento teórico del problema de } \\
\text { investigación. Recomendación de lectura del tema } \\
\text { para próxima clase. }\end{array}$ & $10 \mathrm{~m}$ \\
\hline
\end{tabular}

De acuerdo a la secuencia de actividades y el modelo metodológico, el cuestionario inicial y final se planteó de la siguiente manera:

\section{Tabla 2. Cuestionario inicial}

$$
\begin{aligned}
& \text { ¿Crees que Harley-Davidson, una empresa líder en su segmento y con una } \\
& \text { marca reconocida mundialmente, debe invertir en crecer? ¿Por qué? } \\
& \hline \text { ¿Cómo le dirías a Harley-Davidson que enfocara una decisión de } \\
& \text { crecimiento? ¿A quién crees que consultaría? } \\
& \hline \begin{array}{l}
\text { ¿Sería más importante para Harley-Davidson realizar un planteamiento } \\
\text { general del problema o conocer sus causas más profundas a la hora de } \\
\text { tomar una decisión decrecimiento? }
\end{array}
\end{aligned}
$$

Ciclos de Mejora en el Aula (2020). Experiencias de Innovación Docente de la US Esta obra se distribuye con la licencia Creative Commons 
Si Harley-Davidson contratara a una empresa exterior para evaluar su decisión de crecer en el mercado, ¿Qué criterio sería más importante, el de la empresa o el del investigador? ¿Por qué?

¿Qué clase de información crees que sería más importantes para Harley-Davidson?

Respecto al cuestionario, es importante indicar que a la vista de los resultados que obtuvimos, se decidió eliminar la última pregunta, ya que la información que aportó respecto al conocimiento de los estudiantes fue muy similar a la segunda.

\section{Aplicación del CIMA}

\section{Descripción del desarrollo del CIMA}

A la primera sesión de aplicación del CIMA asistieron 12 alumnos de manera presencial, y 42 de forma telemática. Estaba dedicada a la primera pregunta fundamental del mapa de contenidos "¿Hay acuerdo entre ambas visiones?" Antes de comenzar, aunque en la presentación de la asignatura se les había comentado, hice referencia a la implantación del CIMA, y a la importancia de estos procesos para la mejora de la docencia universitaria, y les solicité participación activa. Del mismo modo que se mostraron predispuestos, y bastante interesados. Traté de transmitir toda la ilusión que llevaba dentro, y creo que resultó. No obstante, a pesar de la ilusión existía por mi parte cierto grado de escepticismo respecto a la implantación del CIMA y de si realmente tendría las consecuencias de mejora respecto a lo que venía haciendo habitualmente. Más que nada pensaba si los alumnos recibirían la propuesta con la predisposición necesaria, y sobre todo si yo sería capaz de implantarla a pesar de las complicadas circunstancias que teníamos.

Ciclos de Mejora en el Aula (2020). Experiencias de Innovación Docente de la US Esta obra se distribuye con la licencia Creative Commons 
Respecto a la primera actividad, la lectura del caso práctico, había sido previamente colgado en la plataforma, y la mayoría de ellos lo habían leído. No obstante, antes de comenzar, planteé la icónica pregunta inicial de “ ¿Os gustan las motos?". Esto sirvió para relajar el ambiente, y por qué no decirlo, a mí también. Dejé un tiempo individual para su lectura, y posteriormente solicité que alguien lo leyera en voz alta. Quería que detectaran las especiales características de la empresa, ya que se trata de un caso poco habitual, donde no existe un problema de rentabilidad o falta de ingresos, sino una posición competitiva dominante y un arraigo importante de la marca. Solicité que fueran diciéndome ideas en voz alta para anotarlas en la pizarra. Estaban comprendiendo la idiosincrática forma que esta empresa tenía y tiene de hacer las cosas, y las particularidades de sus clientes que reflejan en sus productos.

A continuación, dejando en la pizarra por escrito las especiales características de la marca Harley-Davidson que se convertirían en determinantes posteriores para resolver el caso, les pedí que de manera individual trataran de plantear con un listado de ideas como plantearía Harley el problema de fabricar o no más motos, solo con el conocimiento que tenían y con el caso práctico por delante. Se realizó de manera individual para que se enfrentaran en un primer momento al problema antes de exponerlo en grupo. Fui pasando por los sitios aclarando pequeñas dudas sobre el enfoque y observé que encontraron dificultades en el planteamiento de la pregunta, ya que el concepto de problema empresarial apareció demasiado pronto, sin conocer demasiado bien a qué se refería. Anoté en la pizarra el listado de ideas que fueron dándome, dejándolas alli para la siguiente actividad. Además, invité a una alumna a salir a la pizarra para exponer sus ideas. En la actividad de contraste apliqué las ideas que me habían dado y que Ciclos de Mejora en el Aula (2020). Experiencias de Innovación Docente de la US
Esta obra se distribuye con la licencia Creative Commons 
estaban expuestas en la pizarra con el problema del taxi y los VTC, respondiendo a las preguntas expuestas en la secuencia de actividades. La aplicación del caso a otra situación tuvo una gran repercusión y generó bastante atención, y los alumnos se mostraron muy participativos. Hubo bastantes intervenciones, y pude realizar un esquema en la pizarra bastante completo donde relacionaba el caso de Harley con el de las VTC.

Para la fase de ideas, se reunieron en grupo de dos y tres personas en clase, y a través de la plataforma también en casa. Les planteé que en dos columnas indicaran cómo ve el problema la empresa y como lo ve el investigador, de manera que comenzaran con el proceso de dualidad que constituía la base de la primera sesión. Lo realmente interesante aquí fue la oportunidad de pasarme por las mesas y comprobar las opiniones que se iban generando. Comencé a ver que estaban entendiendo los conceptos, y que se mostraban predispuestos hacia la nueva forma. No obstante, encontré ciertas dificultades en la comprensión de problema empresarial, así como en el planteamiento de la actividad. Finalmente, se planteó un esquema-resumen en la pizarra, donde fueron hablando los diferentes grupos de acuerdo a sus aportaciones. La primera sesión concluyó con un ajuste de tiempo bastante aceptable, y con la sensación de que les había gustado. No obstante, las actividades no estaban correctamente expuestas.

En la segunda sesión estuvo dedicada a los componentes del enfoque del problema de investigación (segunda cuestión fundamental del CIMA). Realizamos entre todos un resumen del estado del caso práctico de Harley-Davidson, y de acuerdo a ello les introduje en la segunda parte de la clase, comentándoles la distinción entre Definición-Enfoque-Diseño, y nos separamos en grupo para realizar una ficha de tres columnas sobre los tres aspectos, aplicados al caso de Harley Davidson. Esta aclaración 
realizada por mi parte no estaba prevista en la secuencia de actividades, de manera previa al menos, pero me pareció oportuna debido a las dificultades de comprensión de la actividad que me había encontrado en la sesión anterior. La sorpresa fue cuando fui pasándome por los grupos, y lo que me encontré fue gente contagiada por las ganas de resolver aquello. Las actividades de contraste han tenido desde mi punto de vista más valor en esos paseos y pequeñas charlas grupales donde han descubierto verdaderamente la oposición entre la idea o conocimiento preconcebido ante la pequeña aclaración que se les realizaba.

Tras ello, una alumna salió a la pizarra para anotar sus propias ideas sobre los tres aspectos e ir anotando las diferentes aportaciones de sus compañeros, tanto presenciales como online. La relación directa con otro compañero en la pizarra con un pequeño apoyo por mi parte resultó muy enriquecedora para ellos, ya que se sintieron más tranquilos para responder, así como con una especia de necesidad de ayudar a sus compañeras. Por mi parte, me permitió seguir la clase desde el lado de los alumnos, y fueron realizando comentarios respecto a la materia.

Como actividad de contraste, quise que la cantidad de ideas que tenían hasta el momento la aplicaran a otro caso práctico, como había ocurrido con éxito en la primera sesión, concretamente de McDonald. Comenzamos a leerlo y fueron comparando las diferentes apariciones de elementos del caso. No obstante, ocurrió algo que me hizo desviarme, de manera positiva, de la actividad prevista. Los alumnos comenzaron a aportar nuevas ideas sobre el caso en función de la nueva aclaración de McDonald, y fueron identificando los diferentes aspectos del caso práctico pero aplicados a la nueva versión. Me di cuenta que eran capaces de identificar las principales características del problema en otro supuesto. Creo que les resultó más sencillo incluso, como si estuvieran algo atascados en el caso práctico de Harley. 
En la actividad de contraste traté de realizar un equilibrio entre ambos casos, aprovechando la inercia existente y el interés que había suscitado, reconduciendo la actividad hacia la nueva pregunta, que era respecto a la construcción de un modelo analítico que permitiera una visión de la actividad y del problema de investigación en un solo vistazo. A la vista de las intervenciones por parte de los alumnos aplicando conocimientos relacionales entre ambos casos, consideré oportuno alargar más este punto, y entrar posteriormente en la realización en grupo de un modelo analítico. Sin embargo, ante la falta de tiempo, cerramos la sesión con un breve resumen en la pizarra y se mandó la actividad del modelo analítico para comenzar al día siguiente. La sensación que tuve es que la clase les había sabido a poco, y en parte a mí también.

La tercera sesión comienza con una disposición diferente por parte de los alumnos, ya que venían muy convencidos de la clase anterior, aparte de que tenían encargada una tarea respecto a la realización de un modelo analítico. Sin embargo, algunos alumnos la habían realizado, y otros no, bien por falta de tiempo o por olvido. Así que dejé unos minutos en grupo para realizarla. Quizás un recordatorio respecto a la necesidad de hacer la tarea hubiera sido más recomendable. Antes de la actividad hice un breve resumen de la sesión anterior, relacionando todos los aspectos al caso que estamos resolviendo. La actividad presentó bastantes problemas, quizás porque no comprendieron del todo cuál era el objetivo, o por la falta de capacidad de síntesis para la elaboración de un modelo, situación ya repetida en la sesión anterior, y que merece mi atención para la resolución del problema. La hicieron en grupo. Posteriormente salió una alumna voluntaria a resolverlo, y fuimos comentando las diferentes aportaciones de cada uno. Como contraste expuse artículos científicos reales donde estaban sintetizados los modelos analíticos, de diferentes maneras, y esta actividad sustituyó a la planteada en la secuencia respecto a la lectura de caso real. A continuación, 
como inicio a la siguiente actividad, expuse un video de YouTube con fracasos empresariales de empresas de alto prestigio y poder de mercado, para que vieran como había casos similares de situaciones competitivas privilegiadas y malas decisiones basadas en una investigación de mercados deficiente, y cómo las hipótesis de futuro estaban mal planteadas. Se inició un interesante debate acerca del diseño de problemas de investigación en estas empresas (Kodak, BlackBerry, BlockBuster), siempre con una visión a posteriori, aunque veía que comprendían el concepto inicial. El tiempo pasó bastante rápido, y no quise detener la actividad porque los alumnos comenzaban a adelantar la materia que vendría posteriormente (preguntas de investigación e hipótesis) solo relacionando lo anterior y con la visualización del vídeo. De hecho, un alumno se centró en el caso de BlackBerry, exponiendo de manera clara cuáles debían ser las preguntas de investigación de deberían haberse planteado, por lo que aproveché para escribirlas en la pizarra y realizar una actividad de contraste explicando la diferencia entre éstas y las hipótesis. Esto me sirvió de llave para la siguiente tarea: plantear las preguntas e hipótesis del caso Harley, en grupo, pero el tiempo se había agotado. Lo dejamos para la siguiente sesión, no sin antes realizar un pequeño esquema en la pizarra con las principales ideas de la sesión. La sesión tercera ha sido la que más ha variado respecto a la secuencia de actividades planteada, aunque nunca sin alejarnos del modelo metodológico propuesto.

Como elemento a destacar, al finalizar la sesión un alumno se me acercó al pasillo exterior y me comentó algunos asuntos del caso BlackBerry. Posteriormente, y para mi sorpresa, me pidió que le dijera en qué consistía el CIMA que estábamos realizando, lo cual me causó bastante sorpresa. Le expliqué el cambio en la metodología, la elección de los contenidos esenciales y la necesidad de evaluación, y tras ello, me dio las gracias en nombre de la clase porque estaban disfrutando mucho el nuevo método implantado. Sin duda, ha sido el mejor momento de todo este proceso. 
La cuarta sesión supone una nueva rotación de alumnos, por lo que acuden a clase los que hicieron la primera sesión. Sin embargo, dadas las circunstancias actuales y las medidas por tomar, solo acuden a clase 3 personas, algo que produce en mí una profunda desazón. No obstante, considero que terminar el CIMA de la mejor manera posible es prioritario, aunque los tiempos se reduzcan. Desde casa, solo 26 personas se encuentran conectadas. Realizo un breve resumen de la situación del caso para pasar a la actividad pendiente, el planteamiento de las preguntas de investigación e hipótesis del caso Harley. Propongo que se realicen tres preguntas y tres hipótesis, de manera grupal en clase e individual online, para ponerlas posteriormente en común. Me acerco al grupo y empezamos a construir las cuestiones, algo que se convierte en una clase particular dado el ínfimo número de alumnos, aunque la experiencia es muy enriquecedora, dado que es una circunstancia poco frecuente. Tras ello, los tres alumnos salen a la pizarra a exponer sus cuestiones y sus hipótesis, que complementamos con algunas más provenientes desde casa. Mi miedo a la poca participación se desvanece: son pocos, pero intensos.

La actividad de contraste por mi parte consiste en relacionar las hipótesis y preguntas de investigación con el tipo de dato necesario para obtenerlas, algo que voy complementando con las intervenciones online y presenciales. Así que, como segunda actividad, les entrego a cada uno un folio con dos columnas, siendo la primera un listado de tipo de datos y la segunda una clasificación de los mismos, para que relacionen unos con otros (Anexo 2). Esta actividad la realizaron de forma individual, y al contrario de otras realizadas, si la tuvieron bastante clara. Como contraste, corregimos la actividad en la pizarra con una alumna voluntaria, mientras iba por mi parte aplicando a cada uno de ellos una breve explicación. Tras ello y como nueva actividad, solicité que, de los cuatro conjuntos de datos, indicaran cuáles serían aplicables al modelo de Harley. Para

Ciclos de Mejora en el Aula (2020). Experiencias de Innovación Docente de la US Esta obra se distribuye con la licencia Creative Commons 
mi sorpresa, fueron capaces de sacarlo, y de relacionarlo con el resto de contenido.

Para la solución, expuse el mapa de contenidos completo y fui pregunta por pregunta recopilando las respuestas que ellos mismos habían ido indicando en las sesiones. Se sorprendieron mucho de la supuesta complejidad que le vieron al principio, y como habían ido poco a poco completándolo, y por tanto resolviendo el caso. Mi impresión general fue satisfacción. Y alivio.

\section{Evaluación del aprendizaje de los estudiantes}

Vamos a analizar por motivos de espacio sólo la primera pregunta del cuestionario de evaluación, para conocer la evolución en cuanto al conocimiento de los estudiantes. Esta pregunta del cuestionario de evaluación iba dirigida a captar el nivel de comprensión de los alumnos sobre el caso práctico, ya que era determinante en una situación inicial de la materia. La respuesta, derivada de las aportaciones del caso, es un sí rotundo, que podría desprenderse de la lectura. No obstante, ese sí debía ser matizado en función de los conocimientos que la empresa tuviera de su entorno, y como toda decisión, debía tener un enfoque multidimensional para tomar la decisión adecuada o, al menos, para mitigar la posibilidad de error en la misma.

En el cuestionario inicial, todos los alumnos se situaron en una posición dicotómica, sin especificar u observar la respuesta deseada de una observación general de todos los aspectos del problema. No obstante, tras la implantación del CIMA, se produce un claro desplazamiento hacia la respuesta afirmativa con el $85 \%$ de la clase opinando ya de manera positiva, en incluso casi la mitad, argumentado la respuesta de la multidimensionalidad.

Ciclos de Mejora en el Aula (2020). Experiencias de Innovación Docente de la US Esta obra se distribuye con la licencia Creative Commons 
Pregunta 1: ¿Crees que Harley-Davidson, una empresa líder en su segmento y con una marca reconocida mundialmente, debe invertir en crecer? ¿Por qué?

$\begin{array}{ccccc} & \text { Respuesta 1 } & \text { Respuesta 2 } & \text { Respuesta 3 } & \text { Respuesta } 4 \\ \text { Inicial } & 3 \%(1 \text { alum. }) & 32 \%(12 \text { alum. }) & 68 \%(26 \text { alum.) } & 3 \%(1 \text { alum.) } \\ \text { Final } & 4 \%(1 \text { alum. }) & 11 \%(3 \text { alum. }) & 37 \%(10 \text { alum.) } & 48 \%(13 \text { alum.) }\end{array}$

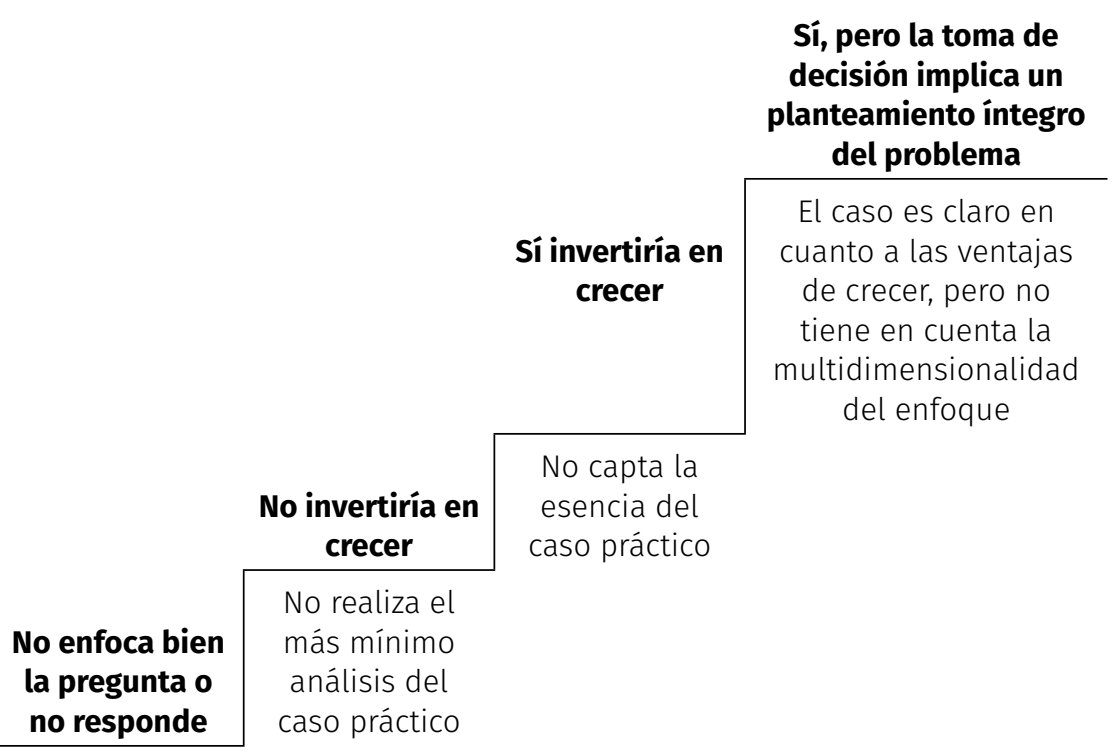

Figura 3. Escalera de aprendizaje para la primera cuestión

Los obstáculos de aprendizaje se situaban principalmente en la propia capacidad de emitir una respuesta, bien sea afirmativa o negativa, aunque pocos alumnos se encontraban en esa situación. La mayor dificultad estuvo en comprender la necesidad de enfocar el problema de manera global, no con una afirmación, y es donde debía incidir con las actividades, sobre todo aquellas que abarcaran aspectos generales de la empresa y otros elementos de decisión globales.

Ciclos de Mejora en el Aula (2020). Experiencias de Innovación Docente de la US Esta obra se distribuye con la licencia Creative Commons 
Tabla 3. Cuadro de evolución para una muestra de alumnos

\begin{tabular}{|l|ccccccccc|}
\hline & \multicolumn{3}{|c}{ Pregunta 1 } & \multicolumn{2}{c}{ Pregunta 2 } & \multicolumn{2}{c}{ Pregunta 3 } & \multicolumn{2}{c|}{ Pregunta 4 } \\
& Inicial & Final & Inicial & Final & Inicial & Final & Inicial & Final \\
\hline Alcalá & 3 & 3 & 4 & 4 & 3 & 4 & 1 & 1 \\
\hline Ángel & 2 & 3 & 3 & 4 & 2 & 3 & 1 & 2 \\
\hline Capkirk & 3 & 3 & 1 & 2 & 4 & 3 & 1 & 1 \\
\hline Cristinamartin & 2 & 2 & 3 & 3 & 2 & 2 & 1 & 3 \\
\hline Diamantes & 3 & 3 & 1 & 1 & 4 & 2 & 1 & 1 \\
\hline El gordo & 3 & 4 & 3 & 4 & 2 & 4 & 1 & 1 \\
\hline Franiplus & 2 & 4 & 3 & 4 & 2 & 1 & 1 & 3 \\
\hline JuanMR & 3 & 4 & 1 & 3 & 2 & 4 & 2 & 3 \\
\hline Lachi & 3 & 4 & 3 & 2 & 1 & 4 & 2 & 3 \\
\hline Lunares & 2 & 4 & 3 & 2 & 1 & 2 & 2 & 3 \\
\hline Mfeito14 & 3 & 4 & 3 & 1 & 3 & 1 & 2 & 1 \\
\hline Paradise & 3 & 3 & 3 & 3 & 4 & 4 & 3 & 1 \\
\hline
\end{tabular}

\section{Evaluación del CIMA}

De manera general, la implantación y desarrollo del CIMA ha funcionado con una eficacia considerable y, sobre todo, con una valoración muy positiva por parte de los alumnos. Sin embargo, este hecho no debe alejarnos de algunas circunstancias para valorar de manera detenida. Si bien el nuevo modelo metodológico ha sido bien recibido por los alumnos, es cierto que las circunstancias han hecho que el nivel de asistentes sea muy bajo y, por tanto, la valoración no puede realizarse en su conjunto. A pesar de los esfuerzos, al final existe una considerable diferencia entre aquellos que reciben la clase online de aquellos que lo hacen de manera presencial, por lo que la implantación del CIMA sufre alteraciones respecto a un desarrollo presencial total. Aun así, los alumnos han recibido de buen grado la implantación del sistema ya que ha

Ciclos de Mejora en el Aula (2020). Experiencias de Innovación Docente de la US Esta obra se distribuye con la licencia Creative Commons 
supuesto una ruptura con lo realizado hasta el momento en la asignatura, en su enfoque y en su metodología, por lo que una ampliación generalizada a todo el temario podría ser conveniente.

El modelo metodológico ha sido desarrollado en todas las sesiones del mismo modo, y se ha cumplido el desarrollo del sistema hipotético-deductivo (Porlán, 2017). No obstante, requeriría más preparación para la vinculación del mapa de contenidos con las actividades. La aplicación del contenido a un caso práctico real despertó la curiosidad e inquietud de los alumnos, cierto, pero esa curiosidad ha hecho que las actividades de contraste orientadas hacia la confrontación de ideas en otros casos prácticos reales derivaran en interesantes debatas dificiles de reorientar hacia el caso original. Por ello, a pesar de la participación en la secuencia de actividades, es posible que la entrega o conocimiento de actividades de manera previa a las sesiones pudiera solucionar este problema.

Los alumnos han recibido de buen grado las actividades de contraste que implicaban interrelación con ellos, ya que generaban un ambiente de confianza que derivaba hacia respuestas con más contenido e implicación en la clase. Sin embargo, para el contenido que impartimos en Investigación Comercial, las actividades de contraste que han supuesto aclaraciones teóricas la atención ha disminuido considerablemente, ya que eran momentos en los que nos acercábamos al modelo metodológico tradicional del que tratábamos de huir. Quizás este problema, cuando se trate de una aclaración imprescindible, pueda ser compensado con actividades que impliquen en la pizarra la participación de los alumnos, de manera que se cree una situación mixta entre la transmisión y la deducción.

Ciclos de Mejora en el Aula (2020). Experiencias de Innovación Docente de la US Esta obra se distribuye con la licencia Creative Commons 
Para mi práctica habitual, considero fundamental la preparación más profunda de las diferentes sesiones, así como la incorporación de un proceso de mejora continua de las mismas mediante la utilización de un diario de sesiones adecuado, actualizado, comprometido y fiable. He sentido una gran presión cuando los contenidos se acababan, o cuando la propia dinámica de la clase me hacía reconducirla hacia el caso con argumentos que deberían estar más preparados, ya que los alumnos lo han notado. Entiendo que han valorado el esfuerzo, pero también han sido conscientes de las faltas que se han cometido por mi parte.

Las preguntas de evaluación han debido de desarrollarse una vez tuviera el mapa de contenidos perfectamente claro pero, aun así, y dada la experiencia con Investigación Comercial, es posible que construyera un cuestionario diferente, con unas preguntas más adaptadas al contenido. sin embargo, la adecuación del cuestionario a un caso práctico acerca de manera más fehaciente, a mi opinión, los mapas mentales de los alumnos en esta asignatura, ya que requiere de un alto grado relacional muy necesario en la disciplina de administración de empresas que cursan.

Desde el punto de vista del contenido, el agobio como docentes que sentimos ante el cumplimiento del temario debe superarse mediante una selección adecuada, de manera individual siguiendo la libertad de cátedra, o de manera colectiva mediante los acuerdos con los coordinadores de las asignaturas. Esa presión llega a hacer que contenidos fundamentales sean tratados como contenidos no esenciales. Una vez finalizado el CIMA, comenté a los alumnos que es posible que encontraran en las transparencias material que no habíamos tocado en el desarrollo del CIMA, y que era materia de evaluación, aunque también les informé de que el contenido fundamental lo 
conocerían como ningún otro grupo de la asignatura. La reacción fue de aceptación ya que habían disfrutado con la impartición tal como lo había hecho yo. Por ello, a partir de ahora en mis sesiones seré mucho más selectivo con el contenido al que se le presta importancia, realizando jerarquizaciones más detalladas del mismo, e incidiendo en los aspectos fundamentales.

En las aulas de Investigación Comercial hay talento, y se ha visto potenciado con la implantación del CIMA y su modelo metodológico. Lo he notado, y lo han notado los alumnos que se han visto partícipes y han querido ser protagonistas de la situación. Ese talento necesita ser proyectado, y es necesario que en mis sesiones incorpore un sistema para hacerlo, pero sobre todo, para no dejar de hacerlo. Si un alumno es feliz en su aula, es mi misión que siga siéndolo, y muchos alumnos durante este CIMA han sido felices en sus clases.

Ciclos de Mejora en el Aula (2020). Experiencias de Innovación Docente de la US Esta obra se distribuye con la licencia Creative Commons 
Palabras clave: Investigación Comercial; grado de administración y dirección de empresas, docencia universitaria, experimentación docente universitaria, aprendizaje basado en problemas.

Keywords: Business research; business administration and management degree, university teaching, university teaching experimentation, problem-based learning.

\section{Referencias bibliográficas}

Bain, K. (2005). Lo que hacen los mejores profesores universitarios. Valencia: Publicaciones Universidad de Valencia.

De Alba-Fernández, N. y Porlán Ariza, R. (2017). La metodología de enseñanza. En R. Porlán (Coord.), Enseñanza universitaria. Cómo mejorarla (pp. 37-54). Madrid: Morata.

Finkel, D. (2008). Dar clases con la boca cerrada. Valencia: Publicaciones Universidad de Valencia.

Mora, F. (2017). Neuroeducación: Solo se puede aprender aquello que se ama. Madrid: Alianza Editorial.

Porlán, R. (Coord.) (2017). Enseñanza universitaria. Cómo mejorarla. Madrid: Morata.

Ciclos de Mejora en el Aula (2020). Experiencias de Innovación Docente de la US Esta obra se distribuye con la licencia Creative Commons 